\title{
OBSERVATION OF THE DECAY $\mathrm{D}^{*+} \rightarrow \mathrm{D}^{\circ} \pi^{+}$
}

G.J. Feldman, I. Peruzzi,** M. Piccolo,**

G.S. Abrams, M.S. Alam, A.M. Boyarski, M. Breidenbach,

J. Dorfan, W. Chinowsky, D. Fryberger, G. Goldhaber,

G. Hanson, J.A. Jaros, A.D. Johnson, J.A. Kadyk, R.R. Larsen,

D. Lïke, $\dagger$ V. Lüth, R.J. Madaras, H.K. Nguyen, $\dagger \dagger$ J.M. Paterson, M.L. Perl,

T.P. Pun, P. Rapidis, B. Richter, R.F. Schwitters,

W. Tanenbaum, G.H. Trilling, J.S. Whitaker, J.E. Wiss

Stanford Linear Accelerator Center, Stanford

University, Stanford, California 94305

and

Lawrence Berkeley Laboratory and Department of Physics

University of California, Berkeley, California 94720

\section{ABSTRACT}

We have observed the decay $\mathrm{D}^{*+} \rightarrow \mathrm{D}^{\mathrm{O}} \pi^{+}$and have measured its $Q$ value to be $5.7 \pm 0.5 \mathrm{MeV}$. The decay width of the $D^{*+}$ is less than $2.0 \mathrm{MeV} / \mathrm{c}^{2}$. The observation of $\mathrm{D}^{*+} \rightarrow\left(\mathrm{K}^{-} \pi^{+}\right) \pi^{+}$but not of $\mathrm{D}^{*+} \rightarrow\left(\mathrm{K}^{+} \pi^{-}\right) \pi^{+}$sets an upper limit on the amount of $\mathrm{D}^{\mathrm{O}}-\overline{\mathrm{D}^{\circ}}$ mixing.

\section{(Submitted to Phys. Rev. Letters)}

*Work supported by the Energy Research and Development Administration. **Permanent address: Laboratori Nazionali, Frascati, Rome, Italy. tFellow of Deutsche Forschungsgemeinschaft. HPermanent address: LPNHE, Université Paris VI, Paris, France. 
When the $\mathrm{D}^{+}$was discovered in $\mathrm{e}^{+} \mathrm{e}^{-}$annihilations at the center-of-mass energy $\left(\mathrm{E}_{\mathrm{Cm}}\right) 4.03 \mathrm{GeV}$, it was noted that it was produced primarily in association with a system of mass $2010 \pm 20 \mathrm{MeV} / \mathrm{c}^{2}{ }^{1}$ This system was widely interpreted to be an excited state of the $\mathrm{D}^{+}$, the $\mathrm{D}^{*+}$, but its mass and width could not be determined precisely nor were any of its decay modes known. In this Letter we report the observation in higher energy $\mathrm{e}^{+} \mathrm{e}^{-}$annihilations of the decay $\mathrm{D}^{*+} \rightarrow \mathrm{D}^{\mathrm{O}} \pi^{+}$and a precise measurement of its Q value.

The data on which this Letter is based come from about 160,000 hadronic events in the $\mathrm{E}_{\mathrm{cm}}$ range 5.0 to $7.8 \mathrm{GeV}$ collected with the SLAC-LBL magnetic detector at SPEAR. ${ }^{2}$ The average $\mathrm{E}_{\mathrm{cm}}$ for these data is $6.8 \mathrm{GeV}$ and the integrated luminosity is $17 \mathrm{pb}^{-1}$.

The analysis techniques are substantially the same as those used previously. ${ }^{1,3}$ Brief1y, each particle in a multihadronic event is assigned a weight proportional to the probability that it is a $\pi, K$, or $p$. These weights are determined from the measured momentum and time-of-flight over a 1.5 to $2.0 \mathrm{~m}$ flight path using a Gaussian time distribution with a 0.35 ns standard deviation. The $\pi-K-p$ weights are normalized so that their sum is unity for each track. All possible combinations of tracks and particle hypotheses are made with each combination weighted by the joint probability that the tracks satisfy the particular particle hypotheses assigned to them. If the $Q$ value of the decay $\mathrm{D}^{*+} \rightarrow \mathrm{D}^{\circ} \pi^{+}$is small, then the momenta of the $\mathrm{D}^{\circ}$ and $\pi^{+}$in the laboratory frame will be roughly proportional to their masses. Since the magnetic detector has low efficiency for the detection of charged particles with momenta less than $100 \mathrm{MeV} / \mathrm{C}$, the $\mathrm{D}^{*+}$ decay cannot 
be readily detected unless the $D^{\circ}$ momentum is about $1.5 \mathrm{GeV} / \mathrm{c}$ or greater. With this in mind we plot in Fig. 1 the invariant mass spectrum for $\mathrm{K}^{{ }^{+}}{ }^{+}$ combinations weighted in the manner explained above with the condition that the $\mathrm{K} \pi$ pair have a momentum greater than $1.5 \mathrm{GeV} / \mathrm{c}$. A $\mathrm{D}^{0}$ peak with $87 \pm 25$ weighted combinations, corresponding to about 250 events, is clearly visible. Its observed rms width of $34 \mathrm{MeV} / \mathrm{c}^{2}$ is consistent with that expected from experimental resolution. (To avoid unwieldy notation, reference to a state will always imply the sum of that state and its charge conjugate state:)

We take $\mathrm{K}^{-}{ }^{+}$combinations from Fig. 1 in the mass range 1820 to 1910 $\mathrm{MeV} / \mathrm{C}^{2}$ to be $\mathrm{D}^{\circ}$ candidates, combine them with an additional pion, and plot the $D \pi-D$ mass difference spectrum in Fig. 2. There is a clear $D^{*+}$ peak in $\mathrm{D}^{\mathrm{O}} \pi^{+}$(i.e. $\mathrm{K}^{\boldsymbol{F}^{+} \pi^{+} \pi^{+}}$) combinations (Fig. $2(\mathrm{a})$ ). There is at most a sma11 signal in $\mathrm{D}^{\circ} \pi^{-}$(i.e. $\mathrm{K}^{\mp} \pi^{+} \pi^{\mp}$ ) combinations (Fig. 2(b)) which is discussed below. The restrictive kinematics of the $\mathrm{D}^{*+}$ decay give it a signal to background advantage over the $\mathrm{D}^{\circ}$ decay of two orders of magnitude, as can be seen from a comparison of Figs. 1 and $2(a)$. Since there is almost no background below the $\mathrm{D}^{*+}$ peak, the probability is high that each event in the peak is real. Thus, in Fig. 3 we gain statistically by replotting with unit weight each event which contributed at least 0.1 unit to Fig. $2 .^{4}$ The observed width of the $D^{*+}$ peak in Fig. $3(a)$ is consistent with the calculated width expected from experimental resolution alone. At the $90 \%$ confidence level the decay (full) width of the $\mathrm{D}^{*+}$ is less than $2.0 \mathrm{MeV} / \mathrm{c}^{2}$.

Using the data from Fig. 3(a), we determine the $\mathrm{D}^{*+}-\mathrm{D}^{0}$ mass difference to be $145.3 \pm 0.5 \mathrm{MeV} / \mathrm{c}^{2}$, or equivalently the $Q$ value of the decay to be $5.7 \pm 0.5 \mathrm{MeV}$. The error is dominated by systematic uncertainties. A1though the kinematics of this decay allow a precise determination of the 
$D^{*+}-D^{0}$ mass difference, the absolute $D^{*+}$ mass cannot be determined from these data any more accurately than the $\mathrm{D}^{0}$ mass is known. From. Ref: 3, the $\mathrm{D}^{\circ}$ mass is $1865 \pm 15 \mathrm{MeV} / \mathrm{c}^{2}$ which implies that the $\mathrm{D}^{*+}$ mass is $2010 \pm 15 \mathrm{MeV} / \mathrm{c}^{2}$.

Making corrections for geometrical, trigger, and time-of-flight inefficiencies and assuming isotropic production, we calculate that

$$
\sigma_{D}^{* \pm} B\left(\frac{D^{*+}+D^{0}{ }^{+}}{D^{*+}+a 11}\right) B\left(\frac{D^{0} \rightarrow \mathrm{K}^{-} \pi^{+}}{D^{0} \rightarrow a 11}\right)=13 \pm 4 \mathrm{pb},
$$

where $\sigma_{D^{* \pm}}$ is the sum of cross sections for the production of $\mathrm{D}^{*+}$ and $D^{*-}$ with momenta greater than $1.6 \mathrm{GeV} / \mathrm{C}$ and the $\mathrm{B}^{\prime} \mathrm{s}$ are the indicated branching fractions. The error includes both statistical and systematic uncertainties; the later are estimated to be 25\%. Comparing Figs. 1 and 2 (a) and making a correction for efficiencies we can also deduce that $25 \pm 9 \%$ of a11 $\mathrm{D}^{\circ}$ 's with momenta greater than $1.5 \mathrm{GeV}$ come from $\mathrm{D}^{*+}$ decay. We now return to a consideration of the possible signal in $D^{\circ} \pi^{-}$ near a mass difference of $145 \mathrm{MeV} / \mathrm{c}^{2}$ (Figs. $2(\mathrm{~b})$ and $3(\mathrm{~b})$ ). These events could be caused by three effects: (1) double misidentification of a $\pi$ as $a K$ and $a K$ as a $\pi, \quad$ (2) $\Delta C=-\Delta S$ decays, which are expected to be suppressed by $\tan ^{4} \theta_{C}\left(\sim 2 \times 10^{-3}\right)$ or (3) $D^{\circ}-\bar{D}^{\circ}$ mixing, a possibility which has received wide speculation in the literature. ${ }^{5}$ To eliminate the first possibility as much as possible, we select the events from Figs. 3(a) and $3(\mathrm{~b})$ within $2.5 \mathrm{MeV} / \mathrm{c}^{2}$ of the center of the peak and require that the ratio of the time-of-flight weights for the chosen combination to that for the combination in which the $K$ and $\pi$ have been interchanged be greater than three. ${ }^{6}$ Twenty-six out of $38 \mathrm{D}^{\circ} \pi^{+}$and 3 out of $11 \mathrm{D}^{\circ} \pi^{-}$events survive. 
These latter 3 events are consistent with coming from backgrounds. We expect 1.4 evefits from uncorrelated combinations of particles and 0.6 events from $\mathrm{K} \pi$ double misidentification. Thus, at the $90 \%$ confidence level, the fraction of the time that a $D^{\circ}$ decays as if it were a $\overline{D^{\circ}}$ (e.g. to $\mathrm{K}^{+} \pi^{-}$instead of $\mathrm{K}^{-} \pi^{+}$) is less than $16 \%$. $^{7}$ 
1. I. Peruzzi et al., Phys. Rev. Lett. 37, 569(1976).

2. J.-E. Augustin et a1., Phys. Rev. Lett. 34, 233(1975);

F. Vannucci et a1., SLAC report number SLAC-PUB-1862 (to be published in Phys. Rev. D).

3. G. Goldhaber et a1., Phys. Rev. Lett. 37, 255(1976).

4. The threshold value of 0.1 units is not critical since backgrounds increase very slowly as the threshold value is decreased.

5. M.K. Gaillard, B.W. Lee, and J.L. Rosner, Rev. Mod. Phys. 47, 277(1975);

R.L. Kingsley, S.B. Treiman, F. Wilczek, and A. Zee, Phys. Rev. D11, $1919(1975)$;

L.B. Okun, V.I. Zakharov, and B.M. Pontecorvo, Lett. Nuovo Cim. 13, $218(1975)$;

A. De Rújula, H. Georgi, and S.L. Glashow, Phys. Rev. Lett. 35, $69(1975)$

A. Pais and S.B. Treiman, Phys. Rev. D12, 2744(1975);

R.L. Kingsley, F. Wilczek, and A. Zee, Phys. Lett. 61B, 295(1976);

K. Kang and J.E. Kim, Phys. Lett. 64B, 93(1976);

R.L. Kingsley, Phys. Letters 63B, 329(1976);

S.L. Glashow and S. Weinberg, Harvard University report HUTP-76/A158 $(1976)$;

E.A. Paschos, BNL report BNL-21870 (1976);

M. Goldhaber and J.L. Rosner, Institute for Advanced Study report number C00-2220-86 (1976).

6. It should be noted that our $\mathrm{D}^{\circ}-\overline{\mathrm{D}}^{\circ}$ discrimination comes from both the time-of-flight information and the requirement used in constructing Figs. 2 and 3 that the observed $K \pi$ mass lie in the range 1820 to $1910 \mathrm{MeV} / \mathrm{c}^{2}$. 
7. A similar upper limit on $D^{\circ}-\overline{D^{\circ}}$ mixing has been deduced from a study of apparent strangeness conservation in events with an identified $\mathrm{D}^{\circ}$ produced in the $\mathrm{E}_{\mathrm{cm}}$ region 3.9 to $4.6 \mathrm{GeV}$. G. Goldhaber, invited talk at the Annual Meeting of the American Physical Society, Chicago, Illinois, February 7-10, 1977. 


\section{FIGURE CAPTIONS}

1. Weighted invariant mass spectrum for $\mathrm{K}^{ \pm} \pi^{\ddagger}$ combinations with momenta greater than $1.5 \mathrm{GeV} / \mathrm{c}$ at $\left\langle\mathrm{E}_{\mathrm{cm}}\right\rangle=6.8 \mathrm{GeV}$.

2. Weighted $D \pi-D$ mass difference spectra for (a) $\mathrm{D}^{\circ} \pi^{+}$and $\overline{\mathrm{D}}^{\mathrm{O}} \pi^{-}$ (i.e. $\mathrm{K}^{\mp^{ \pm} \pi^{ \pm}}$) combinations and (b) $\overline{\mathrm{D}}^{\mathrm{o}} \pi^{+}$and $\mathrm{D}^{\circ} \pi^{-}$(i.e. $\mathrm{K}^{\mp} \pi^{ \pm} \pi^{\mp}$ ) combinations.

3. Unweighted $D \pi-D$ mass difference spectra for (a) $D^{\circ} \pi^{+}$and $\bar{D}^{\circ} \pi^{-}$ (i.e. $\mathrm{k}^{\mp^{ \pm} \pi^{ \pm}}$) combinations and (b) $\overline{\mathrm{D}}^{\mathrm{o}} \pi^{+}$and $\mathrm{D}^{\mathrm{o}} \pi^{-}$(i.e. $\mathrm{K}^{\mp^{+}} \pi^{+}$) combinations. 


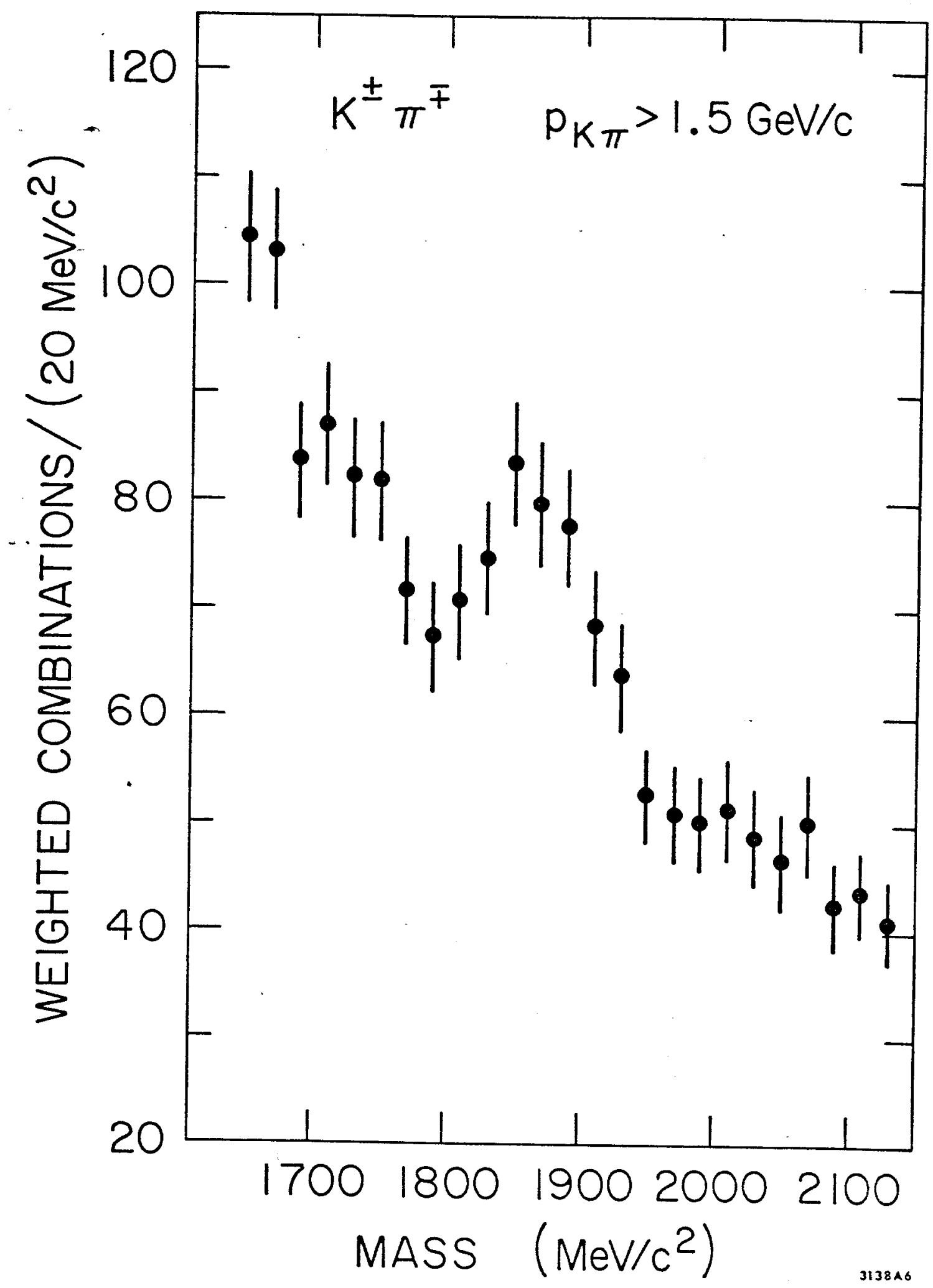

Fig. 1 


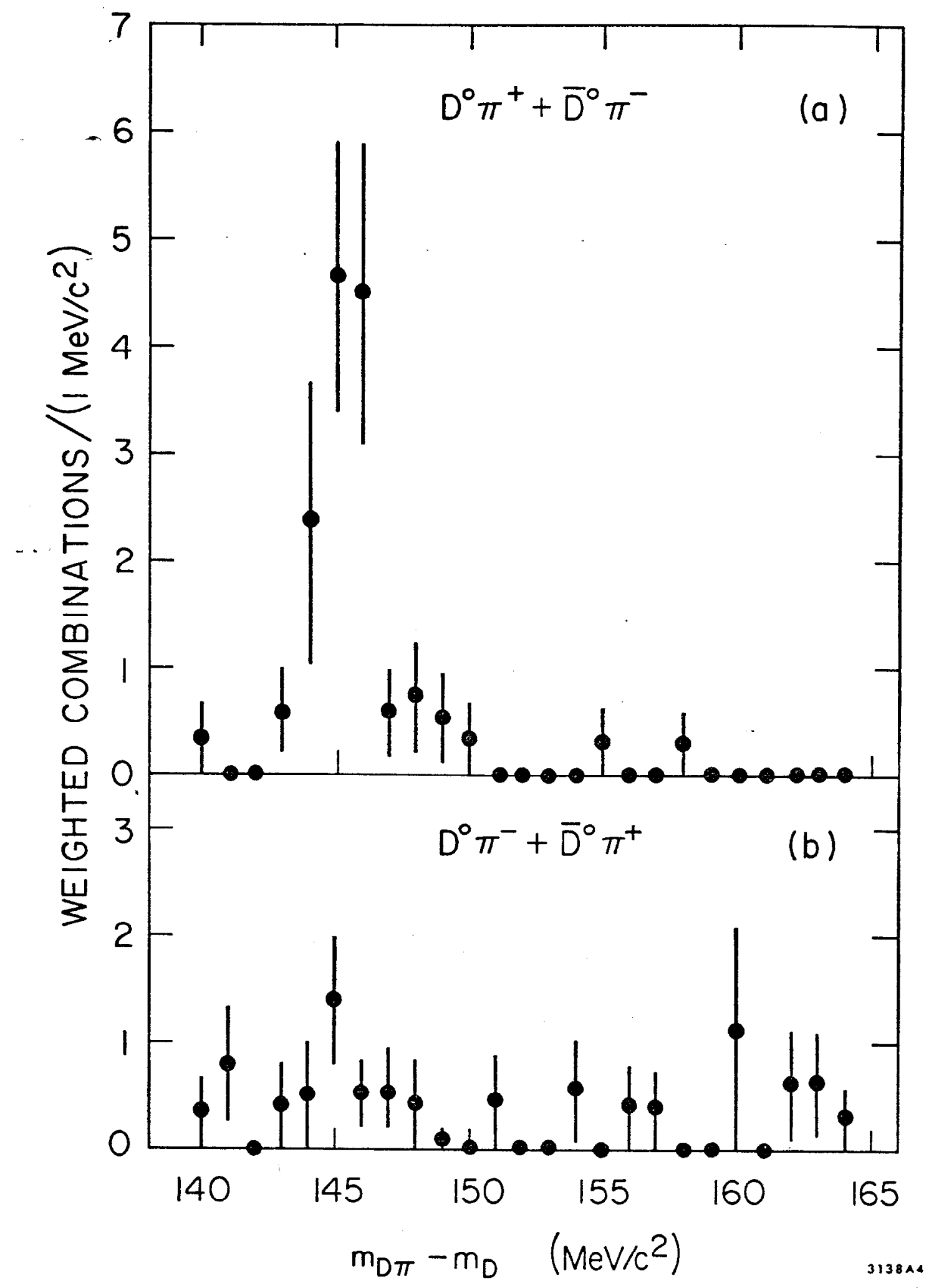

Fig. 2 


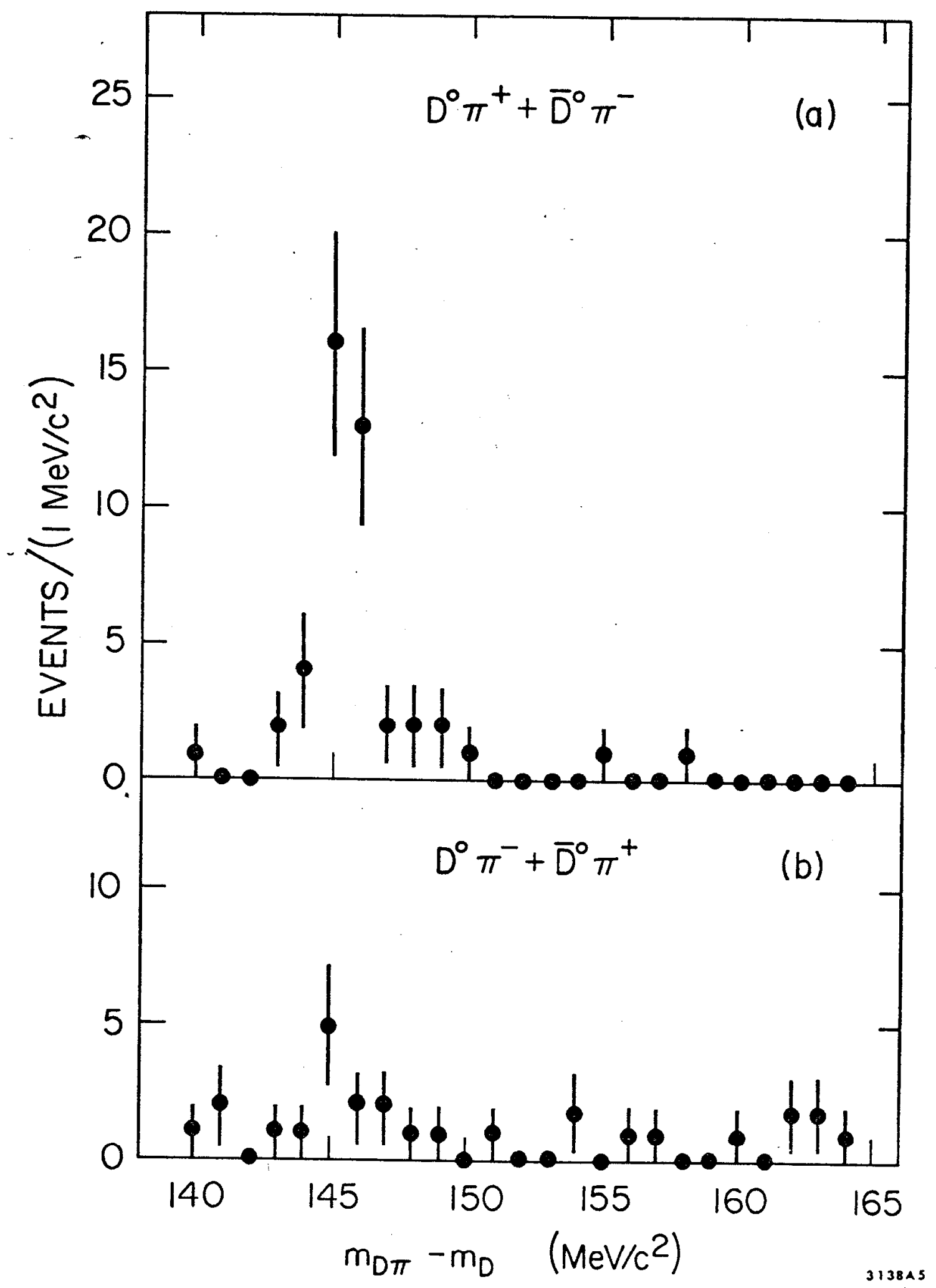

Fig. 3 